\title{
Geochemical Characteristics of Metabasites in Different Tectonic Units of the Northeast Bavarian Crystalline Basement $z$
}

\author{
M. Okrusch ${ }^{1}$, E. Seidel ${ }^{2,3}$, U. Schüssler ${ }^{1}$ and P. Richter ${ }^{1}$
}

\author{
1 Mineralogisches Institut der Universität Würzburg, Am Hubland, D-8700 Würzburg, FRG \\ ${ }^{2}$ Mineralogisches Institut der Technischen Universität Braunschweig, Gauß-Str. 28/29, \\ D-3300 Braunschweig, FRG \\ ${ }^{3}$ Present address: Mineralogisch-Petrographisches Institut der Universität zu Köln, Zülpicher Str. 49, \\ D-5000 Köln, FRG
}

\section{ABSTRACT}

Comprehensive geochemical investigations of metabasites yielded constraints for a correlation of, or discrimination between the different tectonic units within the northeast Bavarian crystalline basement.

The Münchberg nappe pile consists of at least five large tectonic units which exhibit differences in lithology, in part also in metamorphic grade and in metamorphic history. The metabasites in each of these nappes show their own, significant geochemical characteristics. The lowermost tectonic unit, the Bavarian lithofacies, includes the anchimetamorphic Ordovician Randschieferserie which contains alkaline basalts. In their geochemistry, they are similar to the metabasites of the Fichtelgebirge crystalline complex in the autochthonous saxo-thuringian. The next higher tectonic unit of the Munchberg nappe pile, the Prasinit-Phyllit-serie contains metabasites which can be derived from subalkaline basalts with a cleax calc-alkaline tendency. There is a striking geochemical resemblance to the metabasites of the Erbendorf Greenschist zone (EGZ) underscoring the similar lithology of both allochthonous units which appear to be in a similar tectonic position. The Randamphibolit-serie higher up in the Munchberg nappe pile consists of metabasites with tholeiitic characteristics and a pronounced differentiation trend. The next higher tectonic unit, the liegendserie of the Münchberg gneiss complex s. str., contains metagabbros to metagabbronorites with a high-Al basaltic composition. The amphibolites and banded hornblende gneisses of the overlying Hangendserie are of subalkaline basaltic character with calc-alkaline affinity.

The Zone Erbendorf-Vohenstrauss (ZEV) is currently regarded as an allochthonous unit equivalent to the higher crystalline nappes of the Munchberg pile. However, the geochemical character of the metabasites do not encourage such a correlation. Neither the schistose and striped amphibolites nor the flaseramphibolites of the ZEV with their N-MORB and E-MORB character respectively, find convincing counterparts in the crystalline nappes of the Munchberg pile. However, an interestingly close resemblance exists between the schistose and striped amphibolites in the ZEV, on the one hand, and in the autochthonous zone Tirschenreuth-Mähring (ZTM) and the adjacent Moldanubian sensu strictu, on the other.

Owing to the absence of age criteria, our results cannot be used, so far, to reconstruct the paleogeographical position of the individual tectonic units, based on the geochemical characteristics of their respective metabasites. 
During the pre-site studies for the German Continental Deep Drilling Program (KTB) our group investigated the geochemical characteristics of metabasites which form constituents of different tectonic units within the crystalline basement in northeast Bavaria. The aim of these studies was:

1. To evaluate constraints for a correlation and/or discrimination of these tectonic units.

2. To provide a data base for a sound recognition of metabasites and, consequently, their respective units in the future drill cores of RTB.

The geochemical characteristics of the various metabasites may serve as indications for the geotectonic positions of their respective protoliths, provided that the discriminating chemical elements and interelement relationships were not affected seriously by post-magmatic alteration processes.

GEOLOGICAL POSITION AND PETROGRAPHICAL CHARACTERISTICS

The new geotectonic concept developed during the RTB pre-site studies distinguishes two major nappe complexes in the northwestern part of the Bohemian massif:

1. The Muñchberg nappe pile

2. The Zone of Erbendorf-Vohenstrauss (ZEV) with the underlying Erbendorf Greenschist Zone (BGZ).

In contrast, the Saxothuringian of the Frankenwald and the Fichtelgebirge, the Moldanubian and the transitional Zone Tirschenreuth-Măring (ZTM) are regarded as autochthonous (Weber and volibrecht, 1986).

The Münchberg Nappe Pile

The autochthonous Paleozoic of the Thuringian lithofacies in the southeastern Frankenwald is unconformably overlain by a stack of allochthonous units, the Münchberg nappe pile (Franke 1984).

The lowermost nappe unit consists of anchimetamorphic Paleozoic sediments whith intercalations of alkaline basalts to trachyandesites facies by Wurm (1961). keratophyres, designated as Bavarian Iitho-

The next higher unit, the Prasinit-phyllit-Serie (Kraus 1954) consists predominantly of prasinites and quartz phyliites in variable proportions. Locally, serpentinites are intercalated.

The prasinites show the assemblage actinolite + epidote + chlorite + albite + quartz + sphene + opaques typical of the greenschist facies. 
Higher up in the nappe pile a tectonic unit of considerably higher metamorphic grade occurs, the Randamphibolit-serie, which consists almost exclusively of amphibolites (briefly designated as "rand amphibolites") with the assemblage

hornblende + andesine + sphene \pm grossular-rich almandine

\pm epidote + opaques.

The randamphibolites exhibit a strong cataclastic deformation. They underwent a retrograde overprint documented by the formation of actinolitic hornblende, albite, chlorite and epidote (Oppermann 1985).

The next higher crystalline nappe unit is the so-called Liegendserie of the Münchberg Gneiss Complex s. str. Within a sequence of para- and orthogneisses subordinate metagabbros to metagabbronoites (Matthes and Seidel 1977) are intercalated which exhibit distinct structural relics of the plutonic protolith as well as relics of the initial igneous anrich plagioclase, hypersthene, clinopyroxene, brown hornblende, ilmenite and pyrrhotine. The regional metamoxphic overprint led to the assemblage
oligoclase + hornblende
+ rutile/sphene.

The uppermost unit of the Munchberg nappe pile, the Hangendserie, is characterized by a variegated lithology consisting predominantly of amphibolites and banded hornblende gneisses, and subordinate paragneisses, marbles and calc-silicate rocks. The metabasites contain the mineral assemblage

hornblende + oligoclase/andesine + quartz + rutile (partly replaced by sphene) \pm almandine \pm zoisite + opaques.

The banding is due to a variation in the hornblende vs feldspar + quartz ratio.

The famous eclogites of the Munnchberg Gneiss Complex are concentrated in the border zone between the Hangend- and the Liegendserie. They can be interpreted either as constituents of the Hangendserie (Matthes et al. 1974) or as dislodged slices, tectonically intercalated between the two units (stettner 1960). The eclogites testify to a high-pressure event which, according to sm-Nd dating of stosch (pers. comm. 1988), presumably took place $466 \mathrm{Ma}$ ago. The main metamorphic event in the Hangend-, Liegend- and Randamphibolit-Serie is a medium-pressure metamorphism (Matthes et al. 1974; Blümel, in Weber and Vollbrecht 1986) which partly transformed the eclogites into eclogite-amphibolites. Recent $\mathrm{K}-\mathrm{Ar}$ dating on hornblendes and micas from these units yielded ages around $380 \mathrm{Ma}$ for the end of the amphibolite facies metamorphism, whereas the greenschist facies metamorphism of the underlying PrasinitPhyllit-serie seems to be slightly younger, i.e. about 365 Ma (kreuzer et al. 1988).

The Erbendorf-Greenschist Zone (EGZ)

Situated between the autochthonous Fichtelgebirge in the north and the allochtonous zone Erbendorf-Vohenstrauss in the south, the Erbendorf Greenschist zone seems to be in a similar tectonic position as the Prasinit-Phyllit-Serie in the Münchberg nappe pile. The EGz consists mainly of various metabasites, including metagabbros, and of serpentinites, but contains only minor metasediments. The main phase of regional metamorphism took place under conditions of the lowest amphibolite facies leading to the assemblage oligoclase + plagioclase + epidote + opaques. 
During a later retrogressive overprint, the mineral assemblages of this first metamorphic stage were partly replaced by albite, epidote, chlorite, carbonate and quartz. In the contact aureoles of the steinwald and Falkenberg granites, the metamorphic sequences of the EGZ underwent a strong thermal overprint (Matthes 1951; Matthes and Olesch 1986).

The Zone Erbendorf-Vohenstrauss (ZEV)

The 2EV consists predominantly of pelitic metasediments which contain intercalations of various metabasites, ultramafics, calc-silicate rocks, graphite quartzites, graphite schists and orthogneisses. The metabasites can be divided into three main types:

1. Schistose and striped amphibolites prevail in the southern part of the ZEV, but are also recorded in a few outcrops in the northern ZBV. The mineral assemblage is

hornblende + andesine/labradore + sphene + ilmenite + sulphides. The banding is caused by a variation in the hornblende vs plagioclase ratio or by calc-silicate intercalations, mainly consisting of salite + plagioclase \pm garnet.

2. Flaseramphibolites are predominant in the northern and central parts of the ZEV. The flaser structure is caused by ill-defined lenses or wavy bands rich in plagioclase or, rarely. salite. The mineral assemblage is

hornblende + oligoclase/andesine \pm garnet \pm salite + sphene + ilmenite + sulphides.

The garnets are frequently replaced by fine-grained aggregates of plagioclase and hornblendes. At the western margin of the Falkenberg granite, the flaseramphibolites underwent a thermal overprint.

3. Metagabbros are concentrated in the southwestern corner of the ZEV. They are characterized by a coarse-grained, massive structure testifying to a plutonic protolith (Voll 1960). The rocks consist of platy, heavily altered plagioclase and hornblende which is partly replaced by felty aggregates of secondary amphiboles (schüssler 1987). Some metagabbros contain considerable amounts of biotite.

$\mathrm{U}-\mathrm{Pb}$ dating of zircons and monazites (Teufel 1988), and $\mathrm{K}-\mathrm{Ar}$ dating of hornblendes and micas (Kreuzer et al. 1988) from various rock types of the ZEV indicate that the medium-pressure, amphibolite facies metamorphism in the ZEV (Blümel, in Weber and Volibrecht 1986) took place in the Early Devonian, about $380 \mathrm{Ma}$ ago. Younger $\mathrm{K}-\mathrm{Ar}$ ages around $325 \mathrm{Ma}$ which prevail in the eastern $\mathrm{ZEV}$ are presumably a result of contact metamorphism.

The Fichtelgebirge Crystalline Complex

Part of this zone is characterized by a variegated lithology with numerous intercalations of marbles. calc-silicate rocks and minor metabasites ("Bunte Gruppe" of stettner 1975, 1980). There are, however, transitions to a more monotonous lithology with a predominance of pelitic to psammitic metasediments. The sequence underwent a lowpressure metamorphism ranging from the greenschist to the amphibolite facies (Mielke et al. 1979) dated at about 330 Ma by the $k-A r$ method (Rreuzer et al. 1988). Mineral relics of an older medium-pressure event 
were recently recognized by Lenz et al. (in prep.). The regional metamorphic assemblages were partly overprinted by the intrusion of the Fichtelgebirge granites.

Most of the investigated metabasites are fine-grained, schistose amphibolites with the assemblage

hornblende + plagioclase \pm biotite \pm quartz + sphene + ilmenite + sulphides.

Two of the investigated metabasites exhibit relics of an ophitic texture testifying to a doleritic basalt as a protolith. Between the platy plagioclase crystals, there are relics of augite and aggregates of serpentine after primary olivine.

The Zone Tirschenreuth-Māhring (ZTM)

In its lithology the ZTM is less variegated than the Fichtelgebirge crystalline complex, but less monotonous than the Moldanubian gneisses (Richter and stettner 1983). The rare amphibolites of the ZTM and the adjacent Moldanubian s. str. can be hardly distinguished from the schistose and striped amphibolites of the $\mathrm{ZEV}$. The mineral assemblage is hornblende + andesine \pm salite + sphene + opaques.

One amphibolite lense contains garnet which is widely replaced by aggregates of hornblende and plagioclase.

The metamorphism in the ZTM which was dated at about 320 Ma (Teufel 1988; Kreuzer et al. 1988) took place under low-pressure conditions ranging from the greenschist to the high amphibolite facies (Schreyer 1966; Blümel and Wagener-Lohse, in Weber and Vollbrecht 1986).

\section{GEOCHEMISTRY}

Analytical Methods

The major elements Si, Ti, Al, Fetot, Mn, Ca, $\mathrm{K}$ and the trace elements $S, S c, V, C r, C o, N i, C u, Z n, A s, R b, S r, Y, Z r, N b, B a, L a, C e$, Nd were analyzed by standard XRF analysis using lithium tetraborate fusion discs and powder pellets respectively. Mg, Na and $\mathrm{Li}$ were determined by standard AAS methods after decomposing the samples in HF$\mathrm{H}_{2} \mathrm{SO}_{4}$ or $\mathrm{HF}-\mathrm{HClO}_{4}$. P was determined by XRF or spectrophotometrically. Fe (II) by the vanadate method (Peters 1968) or spectrophotometricaliy; $\mathrm{CO}_{2}$ was measured volumetrically, $\mathrm{H}_{2} \mathrm{O}^{+}$by the Penfield method.

In part of the samples $\mathrm{CO}_{2}$ and $\mathrm{S}$ were determined by IR spectrometric methods. W and Mo were analyzed by liquid-liquid extraction with zndithiol (Richter 1984). Rare earth elements were measured by ICP-AES after decomposing the samples in HF-HClO, and separating the REE in chromatographic columns (Erzinger et al. 1984). Selected bulk rock analyses of metabasites from the Munchberg nappe pile, the Erbendorf Greenschist Zone, the Zone Erbendorf-Vohenstrauss, the Fichtelgebirge Crystalline Complex and the zone Tirschenreuth-Mähring are presented in Tables 1 and 2. The REE data for the metabasites of the ZEV, EGZ and ZTM are given in Schüssler et al. (1988). A list of localities and the complete set of geochemical analyses can be obtained from the authors on request. 
rable 1. Selfcted analyses of netabasites fron different tectonic units of the nonchberg nappe pile

\begin{tabular}{|c|c|c|c|c|c|c|c|c|c|c|c|c|c|c|c|c|}
\hline \multirow{3}{*}{$(w t . *)$} & \multicolumn{4}{|c|}{ Prasinit-Phyllit-Serie } & & \multicolumn{5}{|c|}{ Randanphibolit-Serie } & \multicolumn{5}{|c|}{ Engendserie } \\
\hline & $\begin{array}{c}\text { Consond } \\
\text { P11 }\end{array}$ & $\begin{array}{l}\text { Eanples } \\
\text { P22 }\end{array}$ & P26 & P35 & $\begin{array}{c}\text { Hobenk } \\
\text { P38 }\end{array}$ & $\begin{array}{r}\text { Doden } \\
\text { P40 }\end{array}$ & RA16 & RA17 & RA42 & RAs4 & RA23 & $572 / 99$ & $572 / 100$ & 552 & $572 / 108$ & B3 \\
\hline & & & & & & & & & & & & & & & & \\
\hline $\begin{array}{l}\mathbf{s i 0 2} \\
\text { Tio2 } \\
11203\end{array}$ & $\begin{array}{c}18.2 \\
0.67\end{array}$ & $\begin{array}{c}17.4 \\
0.68\end{array}$ & $\begin{array}{c}48.0 \\
1.17 \\
18.3\end{array}$ & $\begin{array}{c}50.1 \\
0.66 \\
17.3\end{array}$ & $\begin{array}{c}17.2 \\
2.28 \\
15.9\end{array}$ & $\begin{array}{c}16.2 \\
1.89 \\
15.6\end{array}$ & $\begin{array}{c}18.5 \\
1.10 \\
14.0\end{array}$ & $\begin{array}{c}47.7 \\
1.91 \\
12.9\end{array}$ & $\begin{array}{c}17.4 \\
1.71 \\
14.8\end{array}$ & $\begin{array}{c}17.4 \\
1.06 \\
14.7\end{array}$ & $\begin{array}{c}50.0 \\
0.53 \\
15.3\end{array}$ & $\begin{array}{c}50.8 \\
0.69 \\
15.9\end{array}$ & $\begin{array}{c}18.4 \\
0.67 \\
16.5\end{array}$ & $\begin{array}{c}52.0 \\
0.64 \\
12.5\end{array}$ & $\begin{array}{c}50.6 \\
0.70 \\
16.5\end{array}$ & $\begin{array}{c}51.9 \\
0.56 \\
15.6\end{array}$ \\
\hline $\begin{array}{l}\text { Te203 } \\
\text { Te0 }\end{array}$ & 3.04 & $\begin{array}{l}1.68 \\
5.56\end{array}$ & $\begin{array}{l}5.77 \\
4.11\end{array}$ & $\begin{array}{l}4.07 \\
3.38\end{array}$ & $\begin{array}{l}3.80 \\
5.83\end{array}$ & $\begin{array}{l}2.77 \\
7.06\end{array}$ & $\begin{array}{l}2.00 \\
9.11\end{array}$ & $\begin{array}{c}1.90 \\
13.4\end{array}$ & $\begin{array}{l}2.00 \\
9.15\end{array}$ & $\begin{array}{l}2.34 \\
6.16\end{array}$ & $\begin{array}{l}1.71 \\
6.25\end{array}$ & $\begin{array}{l}2.09 \\
6.23\end{array}$ & $\begin{array}{l}1.29 \\
7.33\end{array}$ & $\begin{array}{l}3.5 \\
7.85\end{array}$ & $\begin{array}{l}3.7 \\
7.96\end{array}$ & $\begin{array}{r}2.10 \\
8.28\end{array}$ \\
\hline noo & 0.11 & 0.12 & 0.12 & 0.12 & 0.16 & 0.18 & 0.21 & 0.34 & 0.21 & 0.17 & 0.16 & 0.15 & 0.17 & 0.23 & 0.24 & 0.18 \\
\hline $\begin{array}{l}x=0 \\
C=0\end{array}$ & $\begin{array}{r}6.13 \\
13.7\end{array}$ & 13.9 & $\begin{array}{l}5.27 \\
9.50\end{array}$ & $\begin{array}{r}6.52 \\
10.1\end{array}$ & $\begin{array}{l}6.99 \\
8.98\end{array}$ & 8.74 & $\begin{array}{r}8.48 \\
11.8\end{array}$ & $\begin{array}{r}6.69 \\
10.8\end{array}$ & $\begin{array}{r}7.28 \\
12.4\end{array}$ & 10.1 & $\begin{array}{r}8.70 \\
12.7\end{array}$ & $\begin{array}{c}7.92 \\
10.8\end{array}$ & $\begin{array}{r}9.03 \\
12.0\end{array}$ & $\begin{array}{l}9.91 \\
9.26\end{array}$ & $\begin{array}{l}6.11 \\
8.68\end{array}$ & $\begin{array}{l}6.79 \\
7.92\end{array}$ \\
\hline $\begin{array}{l}1020 \\
\times 20\end{array}$ & $\begin{array}{l}1.51 \\
0.49\end{array}$ & 1.36 & $\begin{array}{l}2.82 \\
0.85\end{array}$ & $\begin{array}{l}3.97 \\
0.22\end{array}$ & $\begin{array}{l}3.31 \\
0.96\end{array}$ & $\begin{array}{l}2.83 \\
0.55\end{array}$ & $\begin{array}{l}2.70 \\
0.34\end{array}$ & $\begin{array}{l}1.61 \\
0.16\end{array}$ & $\begin{array}{l}2.24 \\
0.29\end{array}$ & $\begin{array}{l}2.37 \\
0.29\end{array}$ & $\begin{array}{l}2.86 \\
0.26\end{array}$ & $\begin{array}{l}2.88 \\
1.00\end{array}$ & & $\begin{array}{l}2.11 \\
0.21\end{array}$ & $\begin{array}{l}3.19 \\
0.24\end{array}$ & $\begin{array}{l}3.77 \\
0.21\end{array}$ \\
\hline P205 & 0.14 & 0.28 & 0.22 & 0.14 & 0.36 & 0.33 & 0.10 & 0.15 & 0.18 & 0.12 & 0.05 & 0.05 & 0.05 & 0.10 & 0.13 & 0.08 \\
\hline $\begin{array}{l}\mathrm{B2O}+ \\
\mathrm{CO} 2\end{array}$ & $\begin{array}{l}3.0 \\
0.81\end{array}$ & $\begin{array}{l}3.5 \\
0.2\end{array}$ & $\begin{array}{l}3.2 \\
0.1\end{array}$ & $\begin{array}{l}2.8 \\
1.1\end{array}$ & $\begin{array}{l}3.4 \\
0.8\end{array}$ & $\begin{array}{l}3.7 \\
0.7\end{array}$ & $\begin{array}{c}1.9 \\
<0.05\end{array}$ & $\begin{array}{c}1.8 \\
<0.05\end{array}$ & $\begin{array}{l}1.9 \\
0.05\end{array}$ & $\begin{array}{l}2.1 \\
0.06\end{array}$ & $\begin{array}{l}1.6 \\
0.05\end{array}$ & $\begin{array}{l}1.8 \\
0.09\end{array}$ & $\begin{array}{c}1.7 \\
<0.05\end{array}$ & $\begin{array}{l}1.4 \\
<0.05\end{array}$ & $\begin{array}{l}1.2 \\
0.05\end{array}$ & $\begin{array}{l}1.6 \\
0.06\end{array}$ \\
\hline & $<0.02$ & $<0.02$ & 0.02 & 0.17 & 10.02 & 0.02 & $<0.02$ & 80.02 & 0.06 & $<0.02$ & 10.02 & $<0.02$ & 0.11 & 10.02 & 0.22 & 0.11 \\
\hline $\begin{array}{l}\text { Total } \\
\text { (ppa) }\end{array}$ & 99.61 & 99.53 & 99.85 & 100.65 & 99.97 & 99.63 & 100.24 & 99.36 & 99.67 & 100.07 & 100.15 & 100.40 & 99.70 & 9.71 & 9.82 & 99.49 \\
\hline Cr & 161 & 592 & 475 & 205 & 184 & 318 & 376 & 158 & 153 & 811 & 469 & 255 & 300 & 182 & 49 & 83 \\
\hline $\mathbf{m i}$ & 66 & 319 & 137 & 89 & 87 & 152 & 95 & 45 & 71 & 253 & 89 & 148 & 212 & 83 & 30 & 30 \\
\hline cu & 28 & 14 & 56 & 74 & 42 & 83 & 75 & 28 & 115 & 48 & 83 & 19 & 69 & 81 & 290 & 64 \\
\hline $\mathbf{z n}$ & 64 & 5 & 9 & 64 & 80 & 90 & 88 & 129 & 93 & 73 & 62 & 95 & 73 & 92 & 118 & 83 \\
\hline Rb & 10 & 20 & 1 & 5 & 19 & 12 & 8 & 4 & 4 & 9 & 6 & 23 & 16 & 8 & 10 & \\
\hline $3 \mathbf{r}$ & 241 & 412 & 383 & 220 & 351 & 323 & 160 & 58 & 261 & 250 & 168 & 125 & 130 & 93 & 130 & 170 \\
\hline$y$ & 20 & 21 & 31 & 2 & 10 & 36 & 25 & 53 & 28 & 18 & 17 & - & - & 20 & 22 & 15 \\
\hline $2 x$ & 86 & 137 & 111 & 80 & 218 & 239 & 76 & 120 & 122 & 86 & 51 & 55 & 16 & 11 & 38 & 22 \\
\hline ab & 3 & 4 & 3 & 3 & 7 & 8 & 5 & 5 & 13 & 8 & 2 & - & - & $<3$ & 63 & \\
\hline Da & 120 & 192 & 130 & 9 & 210 & 138 & 95 & 79 & 87 & 27 & 64 & 176 & 58 & 85 & 126 & 142 \\
\hline $\mathrm{Pb}$ & 2 & 4 & 5 & \&1 & 3 & 5 & (1) & $\ll 1$ & 3 & (1) & 1 & 1 & 1 & 5 & 4 & $<5$ \\
\hline
\end{tabular}

Table 2. Selected analyses of metabesites from different tectonic units of the xTB target area

\begin{tabular}{|c|c|c|c|c|c|c|c|c|c|c|c|c|c|c|c|c|}
\hline \multirow[b]{2}{*}{$(w t, p)$} & \multicolumn{3}{|c|}{$\begin{array}{l}\text { Zone Erbendorf-Vohens } \\
\text { flaseramphibolites }\end{array}$} & \multicolumn{3}{|c|}{$\begin{array}{l}\text { trauss } \\
\text { schistose striped } \\
\text { anphibolites }\end{array}$} & \multicolumn{3}{|c|}{ netagabbros } & \multicolumn{3}{|c|}{$\begin{array}{l}\text { Erbendort Green- } \\
\text { schist Zode }\end{array}$} & \multicolumn{2}{|c|}{ Fichtelgebirge } & \multicolumn{2}{|c|}{$\begin{array}{l}\text { Zone Tirsched } \\
\text { reuth-ukbring }\end{array}$} \\
\hline & $1-4$ & $2-3$ & $\begin{array}{c}0 P-84 \\
-9\end{array}$ & $\begin{array}{c}O P-84 \\
-36\end{array}$ & $\begin{array}{l}O P-84 \\
-126\end{array}$ & $\begin{array}{l}O P-84 \\
-164\end{array}$ & $\begin{array}{c}O P-84 \\
-72\end{array}$ & $\begin{array}{l}O P-84 \\
-205\end{array}$ & $3-7$ & $\begin{array}{l}\text { RBU-78 } \\
-66\end{array}$ & $\begin{array}{l}\text { RBU }-78 \\
-308\end{array}$ & $\begin{array}{l}\text { RBD }-78 \\
-342\end{array}$ & Tiloa & Wa-1a & 675 & 37 \\
\hline $5 i 02$ & 46.2 & 47.2 & 47.9 & 46.6 & 46.8 & 18.8 & 50.8 & 51.6 & 48.4 & 51.2 & 48.6 & 16.5 & 51.5 & 51.0 & 47.9 & 17.4 \\
\hline $\begin{array}{l}\text { Ti02 } \\
\text { 11203 }\end{array}$ & $\begin{array}{c}1.94 \\
14.5\end{array}$ & $\begin{array}{c}2.12 \\
13.3\end{array}$ & $\begin{array}{r}2.60 \\
15.6\end{array}$ & $\begin{array}{c}1.01 \\
16.2\end{array}$ & $\begin{array}{r}1.29 \\
15.5\end{array}$ & $\begin{array}{l}1.04 \\
15.3\end{array}$ & $\begin{array}{l}1.77 \\
16.2\end{array}$ & $\begin{array}{l}1.85 \\
15.8\end{array}$ & $\begin{array}{c}0.72 \\
14.5\end{array}$ & $\begin{array}{c}0.71 \\
16.4\end{array}$ & $\begin{array}{l}0.58 \\
16.8\end{array}$ & $\begin{array}{c}0.85 \\
18.7\end{array}$ & $\begin{array}{r}3.26 \\
12.4\end{array}$ & $\begin{array}{r}3.92 \\
11.9\end{array}$ & $\begin{array}{l}1.18 \\
14.1\end{array}$ & $\begin{array}{r}1.36 \\
14.9\end{array}$ \\
\hline $\begin{array}{l}r \in 203 \\
T \in 0\end{array}$ & $\begin{array}{l}2.86 \\
8.82\end{array}$ & $\begin{array}{l}2.00 \\
9.70\end{array}$ & $\begin{array}{l}2.86 \\
8.41\end{array}$ & $\begin{array}{l}1.22 \\
8.62\end{array}$ & $\begin{array}{l}1.34 \\
7.88\end{array}$ & $\begin{array}{l}1.66 \\
7.60\end{array}$ & $\begin{array}{l}1.42 \\
8.35\end{array}$ & $\begin{array}{l}1.66 \\
8.22\end{array}$ & $\begin{array}{l}1.17 \\
6.60\end{array}$ & $\begin{array}{r}2.60 \\
5.21\end{array}$ & $\begin{array}{l}3.57 \\
2.43\end{array}$ & $\begin{array}{l}5.28 \\
1.55\end{array}$ & 10.78 & $\begin{array}{l}3.33 \\
10.9\end{array}$ & $\begin{array}{l}0.78 \\
9.73\end{array}$ & $\begin{array}{l}1.13 \\
8.78\end{array}$ \\
\hline Mno & 0.17 & 0.17 & 0.15 & 0.15 & 0.13 & 0.15 & 0.16 & 0.16 & 0.13 & 0.13 & 0.11 & 0.15 & 0.15 & 0.16 & 0.17 & 0.18 \\
\hline ngo & 7.80 & 6.70 & 1.98 & 9.65 & 8.37 & 8.54 & 7.13 & 6.38 & 12.9 & 3.65 & 9.90 & 9.80 & 6.90 & 7.80 & 7.94 & 7.8 \\
\hline $\begin{array}{l}C 20 \\
M=20\end{array}$ & $\begin{array}{c}12.3 \\
2.38\end{array}$ & $\begin{array}{r}13.1 \\
2.45\end{array}$ & $\begin{array}{r}10.9 \\
3.10\end{array}$ & $\begin{array}{c}11.0 \\
3.05\end{array}$ & $\begin{array}{c}12.9 \\
2.61\end{array}$ & $\begin{array}{c}13.0 \\
1.93\end{array}$ & $\begin{array}{l}8.82 \\
2.88\end{array}$ & $\begin{array}{l}9.00 \\
2.81\end{array}$ & $\begin{array}{c}12.6 \\
1.58\end{array}$ & 14.6 & $\begin{array}{c}15.1 \\
0.89\end{array}$ & $\begin{array}{l}8.60 \\
3.45\end{array}$ & 10.0 & 8.65 & $\begin{array}{c}14.2 \\
2.18\end{array}$ & $\begin{array}{c}12.5 \\
1.98\end{array}$ \\
\hline$\times 20$ & 0.18 & 0.58 & 0.98 & 0.20 & 0.15 & 0.13 & 0.65 & 0.72 & 0.22 & 0.27 & 0.23 & 0.38 & 1.02 & 0.10 & 0.16 & 0.13 \\
\hline $\begin{array}{l}8205 \\
720+\end{array}$ & 0.12 & 0.16 & 0.29 & 0.06 & 0.08 & 0.07 & 0.17 & 0.18 & 0.07 & 0.23 & 0.19 & 0.14 & 0.48 & 0.50 & 0.15 & 0.19 \\
\hline $\mathrm{CO} 2$ & $\begin{array}{r}1.0 \\
10.1\end{array}$ & $\begin{array}{r}1.6 \\
<0.1\end{array}$ & $\begin{array}{r}1.3 \\
10.1\end{array}$ & $\begin{array}{r}1.5 \\
10.1\end{array}$ & $\begin{array}{r}1.5 \\
<0.1\end{array}$ & $\begin{array}{r}1.2 \\
80.1\end{array}$ & $\begin{array}{r}1.5 \\
<0.1\end{array}$ & $\begin{array}{r}2.0 \\
<0.1\end{array}$ & $\begin{array}{l}1.2 \\
0.1\end{array}$ & $\begin{array}{l}1.2 \\
0.24\end{array}$ & $\begin{array}{r}2.1 \\
10.1\end{array}$ & $\begin{array}{l}3.9 \\
5.1\end{array}$ & $\begin{array}{r}0.8 \\
10.1\end{array}$ & $\begin{array}{r}2.1 \\
<0.1\end{array}$ & $\begin{array}{r}1.0 \\
<0.1\end{array}$ & $\begin{array}{r}1.9 \\
<0.1\end{array}$ \\
\hline $\begin{array}{l}\text { S } \\
\text { Totel } \\
\text { (ppe) }\end{array}$ & $\begin{array}{r}0.04 \\
99.51\end{array}$ & $\begin{array}{l}10.01 \\
99.38\end{array}$ & 99.17 & 99.26 & 98.55 & 99.42 & 99.85 & 100.38 & 100.19 & $\begin{array}{r}<0.01 \\
100.56\end{array}$ & $\begin{array}{r}0.02 \\
100.52\end{array}$ & $\begin{array}{r}80.01 \\
100.40\end{array}$ & $\begin{array}{l}<0.01 \\
99.96\end{array}$ & $\begin{array}{r}0.13 \\
100.94\end{array}$ & $\begin{array}{r}0.27 \\
99.76\end{array}$ & $\begin{array}{r}0.66 \\
98.91\end{array}$ \\
\hline Li & $<10$ & $(10$ & - & - & - & - & - & - & $<10$ & $<10$ & $<10$ & 33 & 50 & $<10$ & 14 & 15 \\
\hline sc & 38 & 38 & - & - & - & - & - & - & 11 & 30 & 38 & 37 & 33 & 32 & 46 & 47 \\
\hline$\nabla$ & 292 & 324 & 256 & 218 & 222 . & 228 & 240 & 304 & 180 & 150 & 138 & 222 & 350 & 380 & 264 & 276 \\
\hline $\begin{array}{l}\text { Cr } \\
\text { Co }\end{array}$ & $\begin{array}{r}410 \\
57\end{array}$ & $\begin{array}{r}180 \\
16\end{array}$ & 152 & 329 & 378 & 333 & 158 & 156 & 1350 & 380 & 425 & 200 & 100 & 105 & 370 & 380 \\
\hline mi & 205 & 78 & $\begin{array}{r}50 \\
117\end{array}$ & 58 & 58 & 50 & 44 & 41 & 34 & 26 & 24 & 34 & 50 & 66 & 17 & 51 \\
\hline Cu & 36 & 32 & - & 216 & 220 & 97 & 75 & 37 & 250 & 211 & 236 & 192 & 78 & 84 & 135 & 180 \\
\hline $2 n$ & 109 & 201 & - & $\overline{-}$ & - & - & - & - & 60 & 19 & 94 & 16 & 55 & 105 & 57 & 125 \\
\hline As & $<2$ & 8 & $<2$ & $<2$ & - & - & - & - & 56 & 74 & 45 & 62 & 129 & 141 & 116 & 115 \\
\hline $\mathbf{R b}$ & 5 & 9 & 8 & 34 & 3 & 6 & - & - & - & (2) & 12 & $<2$ & $<2$ & 4 & $<2$ & $<2$ \\
\hline $3 x$ & 385 & 385 & 366 & 231 & 266 & 14 & 37 & 16 & 5 & 7 & 7 & 11 & 53 & 5 & 14 & 6 \\
\hline$y$ & 28 & 28 & 28 & 25 & $\begin{array}{r}266 \\
26\end{array}$ & 227 & 302 & 282 & 150 & 412 & 345 & 283 & 790 & 470 & 154 & 144 \\
\hline $\mathbf{z r}$ & 188 & 218 & 187 & 82 & $\begin{array}{r}26 \\
101\end{array}$ & 22 & 32 & 24 & 18 & 20 & 16 & 26 & 29 & 27 & 22 & 28 \\
\hline $\boldsymbol{W b}$ & 20 & 19 & 16 & $<3$ & $\begin{array}{r}101 \\
<3\end{array}$ & 77 & 177 & 106 & 64 & 106 & 64 & 90 & 270 & 264 & 70 & 62 \\
\hline no & 0.58 & 1.18 & - & - & - & -4 & & 6 & $<3$ & 6 & 13 & 5 & 32 & 32 & 5 & \\
\hline Ba & 532 & 382 & 265 & 91 & 55 & 38 & - & - & - & 0.31 & $c 0.10$ & $<0.10$ & 0.36 & 0.91 & $<0.10$ & 0.25 \\
\hline Le & 17 & 17 & - & - & - & 38 & 223 & 210 & 74 & $<5$ & $\langle 5$ & 12 & 228 & 216 & 80 & 65 \\
\hline Ce & & 35 & 22 & $<10$ & $<10$ & - & - & - & 13 & 18 & 9 & 11 & 27 & 26 & - & 8 \\
\hline nd & 21 & 15 & - & - & - & 110 & 21 & 17 & ८10 & 36 & 26 & 18 & 55 & 54 & $<10$ & 15 \\
\hline 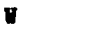 & 0.36 & 0.31 & - & - & - & - & & 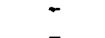 & & 18 & 12 & 12 & 23 & 25 & 19 & 12 \\
\hline & & & & & & & & & - & .65 & 0.34 & 0.10 & 0.66 & 1.32 & 0.28 & $<0.10$ \\
\hline
\end{tabular}




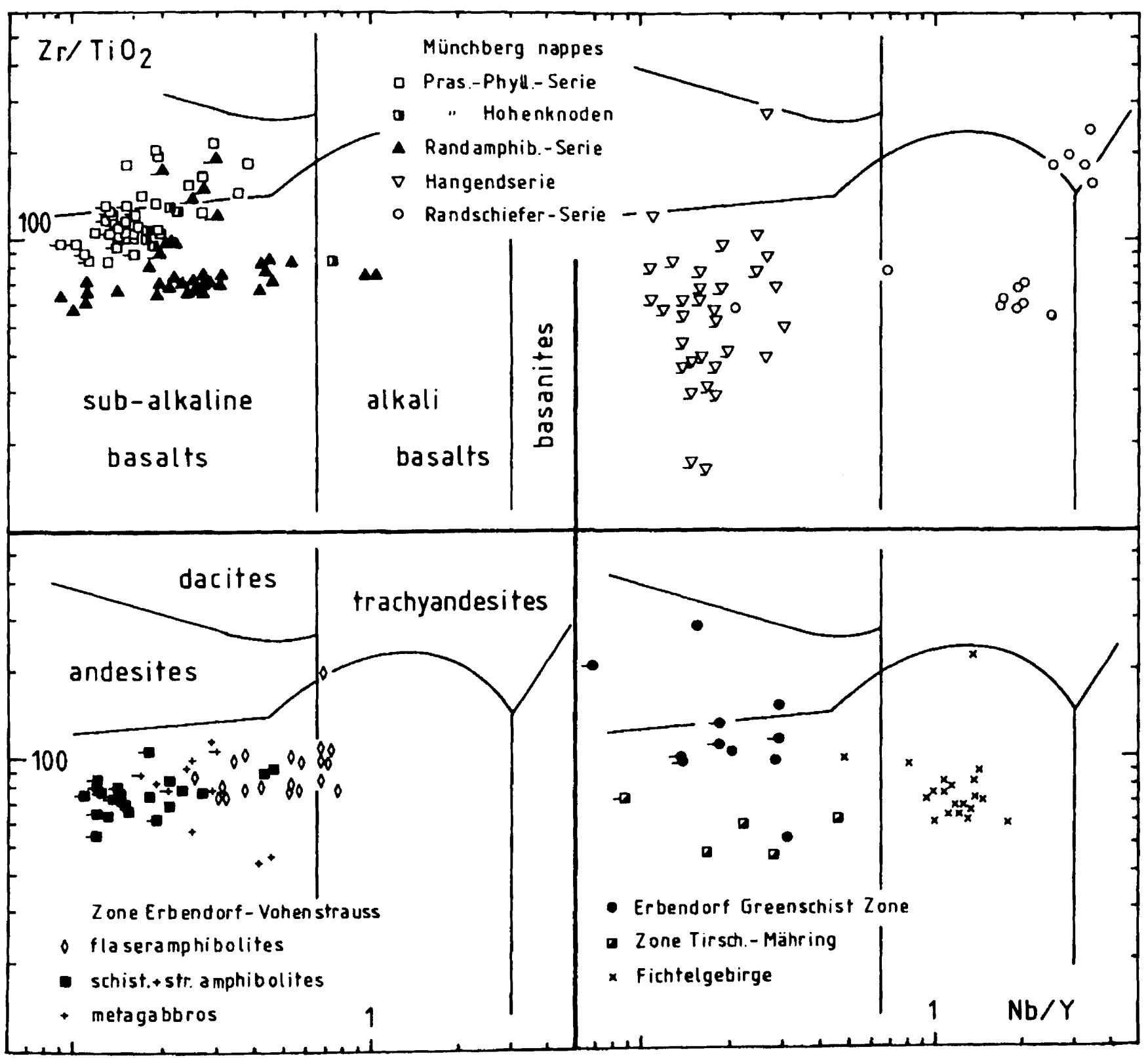

Fig. 1. Discrimination between subalkaline and alkaline series by $\mathrm{Nb} / \mathrm{Y}$ vs $\mathrm{zr} / \mathrm{TiO}_{2}$ (Floyd and Winchester 1978). Samples with Nb-contents below the detection limit of $3 \mathrm{ppm}$ are indicated by bars. $\mathrm{TiO}_{2}$ in wt. $\%, \mathrm{Nb}, \mathrm{Zr}, \mathrm{Y}$ in $\mathrm{ppm}$

The Mūnchberg Nappe Pile

Because of significant chemical differences, the metabasites in the individual tectonic units of the Münchberg nappe pile cannot be derived from one common type of protolith. This finding clearly contradicts former assumptions according to which the metabasites in the PrasinitPhyllit-Serie, in the Randamphibolit-serie and in the Hangend-Serie are metamorphosed equivalents of the Ordovician alkaline basalts in the anchimetamorphic Randschiefer-Serie of the Bavarian lithofacies. According to their chemical composition (wirth 1978), these ordovian basalts form two different groups of alkaline basaltic and trachyandesitic character respectively (Figs. 1, 3). 

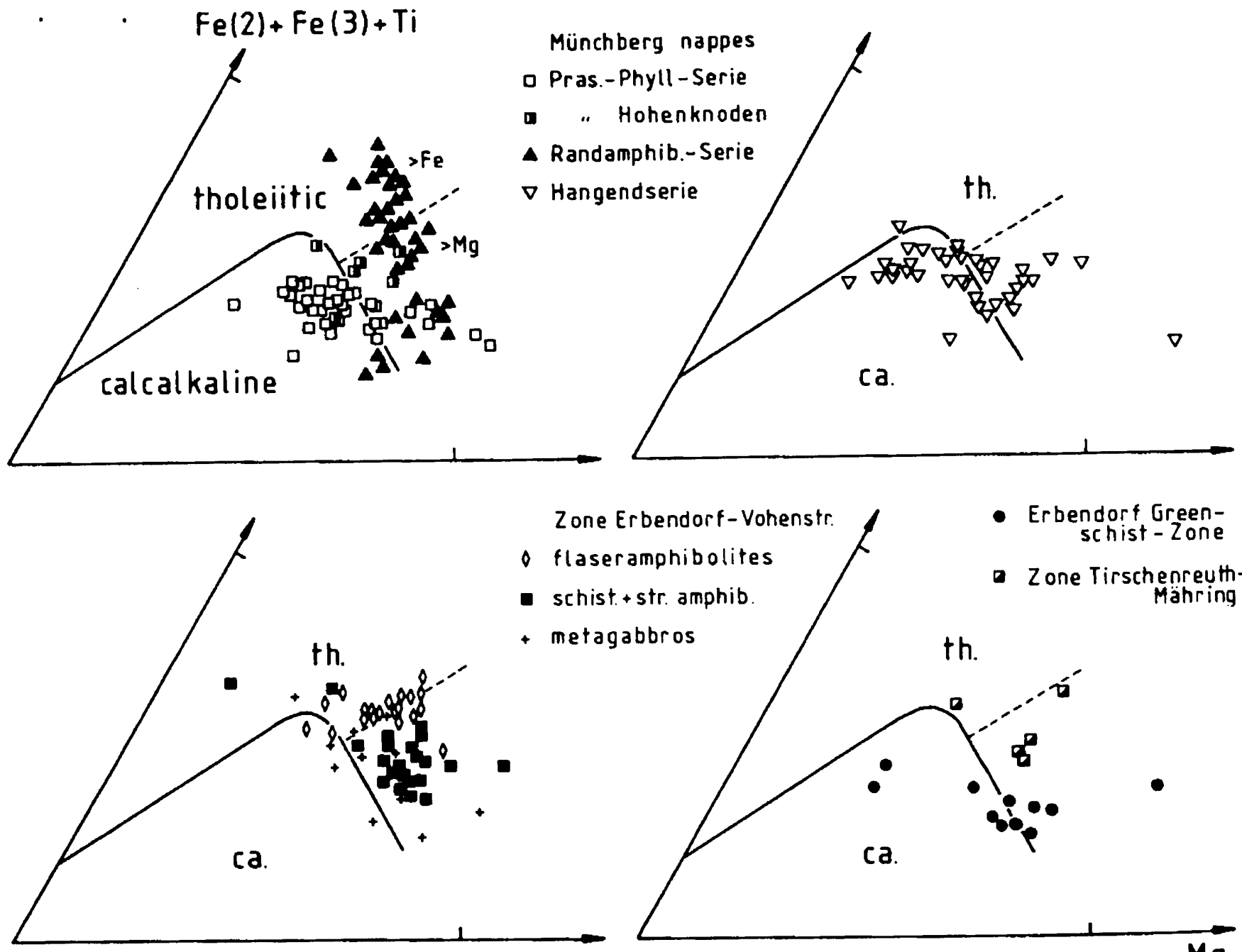

- Erbendorf Greenschist-Zone

- Zone TirschenreuthMähring

Al

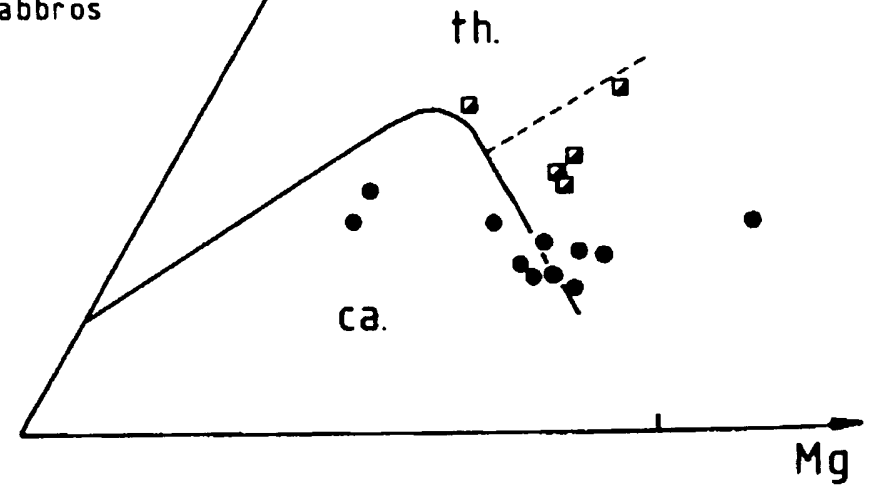

Fig. 2. Cationplot of Jensen (1976) used to subdivide subalkaline basalts into tholeiitic and calc-alkaline series respectively

Judging from their bulk rock chemistry as well as from structural and mineral relics, the metabasites of the Prasinit-phyllit-serie are clearly derived from a basaltic protolith including pyroclastics (opper mann 1985). They are characterized by constantly low TiOz contents (mean value $0.9 \pm 0.2$ wt.\%), but variable $\mathrm{P}_{2} \mathrm{O}_{3}$ and $\mathrm{zr}$ values (Fig. 3). The prasinites are clearly subalkaline in their geochemical character (Fig. 1) and show a calc-alkaline tendency (Figs. 2 , 3).

Prasinites of the abandoned quarry north of Hohenknoden, situated at the tectonic contact between the Prasinit-Phyllit-Serie and the Randamphibolit-serie have distinctly higher values of $\mathrm{TiO}_{2}$ and $\mathrm{Zr}$ than the bulk of the prasinites (Fig. 3). However, the $\mathrm{Zr} / \mathrm{TiO}$ and $\mathrm{Nb} / \mathrm{Y}$ ratios in the Hohenknoden prasinites are the same as in the other prasinites (Fig. 1). Mineralogically the Hohenknoden prasinites are distinguished from the other, sphene-bearing prasinites by the presence of rutile which is more or less replaced by sphene. From the metabasites of the overlying Randamphibolit-Serie the Hohenknoden prasinites are also distinguished chemically. As a consequence we assume that an additional tectonic slice is exposed in the Hohenknoden quarry. 
The amphibolites of the Randamphibolit-Serie conform to subalkaline basalts in their chemical composition. with tholeiitic characteristics (Figs. 1, 2). In contrast to the fairly homogeneous group of the prasinites (disregarding the Hohenknoden samples), the randamphibolites show a pronounced differentiation trend from $\mathrm{Mg}-\mathrm{rich}$ to Fe-rich compositions (Fig. 2). This trend is matched by a parallel enrichment of $\mathrm{TiO}_{2}, \mathrm{Zr}$ (Fig. 3), $\mathrm{P}_{2} \mathrm{O}_{0}$ and $\mathrm{Y}$ and by a depletion of $\mathrm{Ni}$. The geochemical variation is expressed in the mineralogical composition, too: amphibolites with higher $\mathrm{Mg} / \mathrm{Fe}$ ratios are free of garnet, whereas Fe-enriched amphibolites usually are garnet-bearing (Oppermann 1985).

In their bulk rock chemistry, the metagabbros to metagabbronorites of the Liegendserie conform to a high-Al basaltic composition, similar to that of the light eclogite type in the Hangendserie. However, judging from differences in e.g. the $\mathrm{Zr}$ and $\mathrm{Ti}$ contents, Matthes and seidel (1977) deny a genetic relationship between these two rock types.

The amphibolites and banded hornblende gneisses of the Hangendserie are derived from a basaltic protolith (Pommerenke 1985) with consistently low $\mathrm{TiO}_{2}$ and $\mathrm{Zr}$ contents (Fig. 3). They belong to the subalkaline group (Fig. 1) with a calc-alkaline trend (Fig. 2). In the relevant correlation diagrams the metabasites of the Hangendserie show an affinity to those of the Prasinit-Phyllit-serie, but have usually lower $\mathrm{zr}$ contents. The chemical differences to the tholeiitic Randamphibolites and the alkaline basalts of the anchimetamorphic Ordovician Randschieferserie (Bavarian lithofacies) are obvious (Figs. 1, 2, 3).

The eclogites of the Munchberg Gneis Complex can be divided into two principal groups: The dark eclogites have an N-MORB like composition, whereas the light, kyanite-bearing eclogites are high-Al basaltic in character (Matthes et al. 1974; Puchelt et al. 1978) and may be derived from plagioclase-rich, gabbroic cumulates (stosch and Lugmair 1987). Despite some geochemical similarities, the light eclogites cannot be derived from the metagabbros and metagabbronorites of the Liegendserie (Matthes and seidel 1977).

The Erbendorf Greenschist Zone (EGZ)

The predominant striped amphibolites and the subordinate metagabbros of the EGZ cannot be distinguished by their chemical compositions which conform to Mg-rich tholeiites to calc-alkaline basalts (Figs. 1, 2). In diagrams related to the tectonic setting of modern basalts the EGZ metabasites plot along the borderline between the midocean ridge and the island arc basalts or into the field of the island arc basalts (Fig. 3). The major and trace element contents of the metabasites in the EGZ and in the Prasinit-Phyllit-serie of the Munchberg nappe pile agree strikingly well. This fact underscores the similar lithological assemblages of both tectonic units.

\section{The Zone Erbendorf-Vohenstrauss (ZEV)}

The schistose and striped amphibolites of the ZEV conform in their chemical composition to basalts of the subalkaline series (Fig. 1). Their Mg-rich tholeitic character is shown in the Jensen cationplot (Fig. 2). Trace elements including REE indicate similarities to modern N-MORB compositions (Fig. 3 ). 


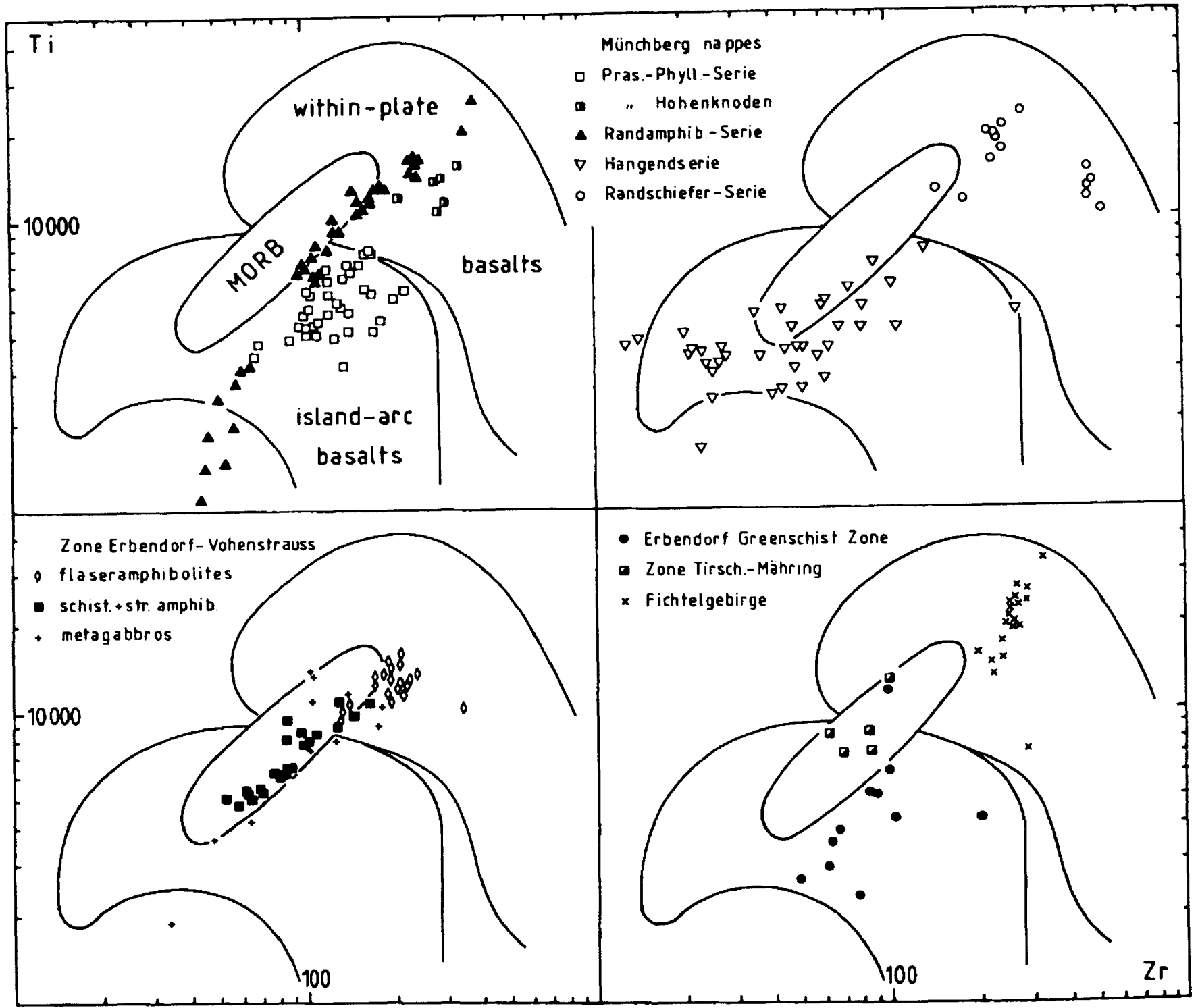

Fig. 3. Comparison of the investigated metabasites with modern basalts from various geotectonic positions in the $\mathrm{Ti}$ (ppm) vs $\mathrm{Zr}$ (ppm) diagramm (Pearce 1982)

In contrast, the flaseramphibolites of the ZEV are clearly enriched in the incompatible trace elements $\mathrm{Nb}, \mathrm{Ce}, \mathrm{P}, \mathrm{Zr}, \mathrm{Ti}$ and the LREB pointing to a tholeiitic protolith with an E-MORB or within-plate basalt character (Fig. 3). Compared to the schistose and striped amphibolites, the often garnet-bearing flaseramphibolites have generally a higher Fe/Mg ratio (Fig. 2). It should be noted that both amphibolite types show a relatively well-defined regional distribution within the ZEV. He, therefore, do not assume a differentiation relationship between the respective protoliths of the two types. This is in contrast to the situation supposed for the randamphibolites of the Munchberg nappe pile.

The metagabbros in the southwest corner of the ZEV are transitional in their geochemical character between the schistose and striped amphibolites and the flaseramphibolites. However, three of the investigated samples (e.g. 3-7 in Table 2) are clearly depleted in incompatible elements and, conformably, have lower $\mathrm{Fe} / \mathrm{Mg}$ ratios and higher $\mathrm{Cr}$ contents (Figs. 2, 3), indicating a more primitive type of gabbroic protolith. 
Some of the metabasites in the $\mathrm{ZEV}$ are chemically influenced by the intrusion of the Leuchtenberg and Falkenberg granites and/or altered by hydrothermal fluids (Schũssler et al. 1988, Schūssler 1989).

The Fichtelgebirge Crystalline Complex

The metabasites of the Fichtelgebirge form a distinct group which is of alkaline basaltic character (Fig. 1). Modern basalts extruded within plates or at anomalous midocean ridges are similar in composition (Fig. 3). The enrichment of incompatible elements is still stronger than in the flaseramphibolites of the northern ZEV (Fig. 3). The Fichtelgebirge metabasites have no geochemical counterpart in the crystalline basement of northeast Bavaria. However, they agree quite well with the alkalinebasalts of the anchimetamorphic Randschieferserie (Bavarian 1ithofacies) in the Münchberg nappe pile.

Zone Tirschenreuth-Māhring (ZTM)

The textural similarities between the schistose and striped amphibolites of the ZEV, on the one hand, and of the ZTM and the adjacent Moldanubian s. str., on the other, are matched by a nearly identical geochemical character, conforming to typical N-MORB compositions (Figs. $1,2,3)$.

\section{CONCLUSIONS}

Metabasites in each of the individual tectonic units of the Munchberg nappe pile exhibit their own, significant geochemical characteristics. This fact indicates that the respective basaltic protoliths were initially emplaced in different paleogeographial environments. Owing to the absence of age criteria, the relative position of these environments in time and space, prior to the stacking of the Munchberg nappe pile, is so far unknown.

The close geochemical resemblance between the metabasites from the Prasinit-Phyllit-Serie of the Munchberg nappe pile and the Erbendorf Greenschist-zone is a strong argument for a correlation of the two units which exhibit a similar lithological association and appear to be in an analogous tectonic position.

In contrast, a correlation between the higher, crystalline nappes in the Munchberg pile and the Zone Erbendorf-Vohenstrauss is not encouraged by our results. No geochemical relationship exists between the amphibolites and banded hornblende gneisses of the Hangendserie and the various metabasite types of the $\mathrm{ZEV}$. The randamphibolites, too, find no convincing counterpart in the $\mathrm{ZEV}$. As a consequence we must state that a connection between the higher units of the Münchberg nappe pile and the ZEV cannot be maintained in the straightforward way which has been postulated recently (weber and volibrecht 1986, p. 26). 
However: an interestingly close correlation exists between the schistose and striped amphibolites of the Zone Erbendorf-Vohenstrauss, on the one hand, and of the Zone Tirschenreuth-Mähring and the adjacent Moldanubian s. str., on the other. The interpretation of this finding is still open to discussion. Geochemical investigations on metabasites from the Zone Tepla-Domazlice and from the Moldanubian s. str. are in progress and hopefully will provide additional constraints.

The geochemical affinity between the metabasites of the Fichtelgebirge crystalline complex and the alkaline basalts of the anchimetamorphic Ordovician Randschieferserie (Bavarian lithofacies) in the lowermost position of the Münchberg nappe pile should be stressed. However, it would be daring to derive an age relationship from this fact alone.

Acknowledgements. Thanks are due to Ms. Rosemarie Baur (Würzburg), otto Ewald, Uwe Oppermann, Rurt Pommerenke (Braunschweig), Frank Volker (Rarlsruhe), Julian Lodziak, Detlef Requard (Hannover), Jörg Erzinger and Evelyn Zuleger (Gießen) for their assistance in the analytical work. The financial support of Deutsche Forschungsgemeinschaft (grant ok 2/25 -1 - Ok 2/25-2) is gratefully acknowledged.

\section{REFERENCES}

Erzinger J, Heinschild H-J, Stroh A (1984) Bestimmung der seltenen Erden in Gesteinen mit der ICP-AES. In: Welz B (ed) Fortschritte in der atomspektrometrischen spurenanalytik, 1. Verlag Chemie, Weinheim, pp 251-260

Floyd PA, Winchester JA (1978) Identification and discrimination of altered and metamorphosed volcanic rocks using immobile elements. Chem Geol 21: 291-306

Franke W (1984) Variszischer Deckenbau im Raume der Mūnchberger Gneismasse - abgeleitet aus der Fazies, Deformation und Metamorphose im umgebenden Paläozoikum. Geotekt Forsch 68: 1-253

Jensen LS (1976) A new cation plot for classifying subalkalic volcanic rocks. Ontario Div Mines MP 66: 1-22

Rraus L (1954) Die geologische Stellung der Prasinit-Phyllit-serie in der Umrandung der Münchberger Gneismasse. Diss, Univ Würzburg 84 pp (unpublished)

Rreuzer H, Seidel E, Schūssler U, Okrusch M, Lenz R-L, Raschka H (1988) $\mathrm{K}-\mathrm{Ar}$ geochronology of different tectonic units at the northwestern margin of the Bohemian massif. Tectonophysics (in press)

Matthes $S$ (1951) Die kontaktmetamorphe oberprägung basischer kristalliner Schiefer im Rontakthof des Steinwald-Granits nordlich von Erbendorf in der bayerischen Oberpfalz. Neues Jahrb Mineral Abh $82: 1-92$

Matthes S, Olesch M (1986) Polymetamorphic-metasomatic blackwall rocks of the Falkenberg granite contact aureole near Erbendorf, oberpfalz, Bavaria. Neues Jahrb Mineral Abh 153: 325-362

Matthes S, Seidel E (1977) Die Eklogitvorkommen des kristallinen Grundgebirges in NE-Bayern. X. Bestehen genetische Beziehungen zwischen Eklogit und Meta-Gabbro innerhalb des Münchberger Gneisgebietes? Neues Jahrb Mineral Abh 129: 269-291

Matthes S, Richter P, Schmidt R (1974) Die Eklogitvorkommen des kristallinen Grundgebirges in NE-Bayern. VII. Ergebnisse aus einer Rernbohrung durch den Eklogitkörper des Weißensteins. Neues Jahrb Mineral Abh 120: 270-314 
Mièlke $\dot{H}$, Brūmel P, Langer K (1979) Regional low-pressure metamorphism of low and medium grade in metapelites and -psammites of the Fichtelgebirge area, NE-Bavaria. Neues Jahrb Miner Abh 137: 83-112

oppermann U (1985) Geochemie und Petrographie der Prasinite und Amphibolite am SW-Rand der Münchberger Gneismasse, NE-Bayern. Dipl-Arb, Tech Univ Braunschweig, $140 \mathrm{pp}$ (unpublished)

Pearce JA (1982) Trace element characteristics of lavas from destructive plate boundaries. In: Thorpe RS (ed) Andesites. Wiley, New York, pp 525-548

Peters A (1968) Ein neues Verfahren zur Bestimmung von Eisen(II) oxid in Mineralen und Gesteinen. Neues Jahrb Mineral Monatsh 1968: 209-214

Pommerenke $R$ (1985) Geochemie und Petrographie der Amphibolite im NETeil der Mũnchberger Gneismasse, NE-Bayern. Dipl-Arb, Tech Univ Braunschweig, $135 \mathrm{pp}$ (unpublished)

Puchelt $H$, Matthes S, Richter P (1978) Aussagemöglichkeiten der seltenen Erden und anderer Spurenelemente für die Eklogite des Münchberger Gneisgebietes. Fortschr Mineral 56: 106-108

Richter P (1984) Wolfram in Graniten Ostbayerns - Versuch einer metallogenetischen Gliederung. Geol Jahrb D 63: 3-22

Richter P, stettner G (1983) Das Prākambrium am Nordrand der Moldanubischen Region im Raum Tirschenreuth-Mähring (NE-Bayern) und dessen metallogenetische Aspekte. Geol Jahrb D 61: 23-91

Schreyer $W(1966)$ Metamorpher Obergang Saxothuringikum-Moldanubikum östlich Tirschenreuth/Oberpfalz, nachgewiesen durch phasenpetrologische Analyse. Geol Rundsch 55: 491-509

Schūssler U (1987) Petrographie, Geochemie und Metamorphosealter von Metabasiten im RTB-Zielgebiet Oberpfalz, Ostbayern. Diss, Univ wūrzburg, $272 \mathrm{pp}$

schūssler U (1989) Petrographie, Geochemie und Metamorphosealter von Metabasiten im KTB-Zielgebiet oberpfalz. Geologica Bavarica (in press)

Schūssler U, Richter P, Okrusch M (1988) Metabasites from the RTB target area Oberpfalz, Bavaria - geochemical characteristics and examples for mobile behaviour of "immobile" elements. Tectonophysics (in press)

stettner $G$ (1960) Ober Bau und Entwicklung der Münchberger Gneismasse. Geol Rundsch 49: 350-375

stettner G (1975) Zur geologisch-tektonischen Entwicklung des oberpfälzer Grundgebirges. Aufschluß Sonderband 26: 11-38

stettner G (1980) Zum geologischen Aufbau des Fichtelgebirges. Aufschluß 31: 391-403

stosch H-G, Lugmaix GW (1987) Geochronology and geochemistry of eclogites from the Munchberg Gneiss Massif, FRG. Terra Cognita 7: 163

Teufel $S$ (1988) Vergleichende U-Pb- und Rb-Sr-Altersbestimmungen an Gesteinen des Obergangsbereichs Saxothuringikum/Moldanubikum, NEBayern. Göttinger Arb. Geol. Paläont. 35: 1-87

Voll G (1960) stoff, Bau und Alter der Grenzzone Moldanubikum/Saxothuringikum in Bayern unter besonderer Berücksichtigung gabbroider. amphibolitischer und kalksilikatführender Gesteine. Geol Jahrb Beih 42: $1-382$

Weber $R$, Vollbrecht A (1986) Rontinentales Tiefbohrprogramm der Bundesrepublik Deutschland, RTB - Ergebnisse der Vorerkundungsarbeiten, Lokation Oberpfalz. 2. KTB-Kolloq, 19.9.-21.9.1986, Seeheim/ Odenwald, 186 pp

Wirth R (1978) Geochemie und Petrographie der paläozoischen Magmatite des Frankenwaldes. Diabase, Reratophyre, Pikrite. Diss, Univ Würzburg, $130 \mathrm{pp}$ (unpublished)

Wurm A (1961) Geologie von Bayern. Borntraeger, Berlin, 555 pp 